\title{
HepciFer study: Hepcidin overexpression after hepatectomy : Does iron supplementation make sense ?
}

\author{
Benoliel A'., Assouline B. ${ }^{2}$, Legouis D. ${ }^{2}$, Blaudzun G. ${ }^{2}$ Kraiem A. ${ }^{2}$, Favre M. ${ }^{2}$, Schiffer E. ${ }^{2}$ \\ I Hôpital Cochin, Dept of Anaesthesiology \& Intensive Care, Paris, France, \\ 2Hôpitaux Universitaires Geneva (HUG), Dept of Anaesthesiology, Geneva, Switzerland
}

\section{Background and goal of the study}

Anemia is frequent in liver surgery with an estimated incidence of $32 \%(1)$. The causes of anemia in surgical patients are often the iron deficiency anemia and the functional iron deficiency (FID). FID is associated with an elevated level of hepcidin (synthetized by the liver), the central regulatory protein of iron metabolism. Our hypothesis was that Hepcidin is overexpressed after liver surgery and contributes to postoperative anemia. We aimed to assess the impact of intravenous iron administration in the immediate postoperative period on hemoglobin levels.
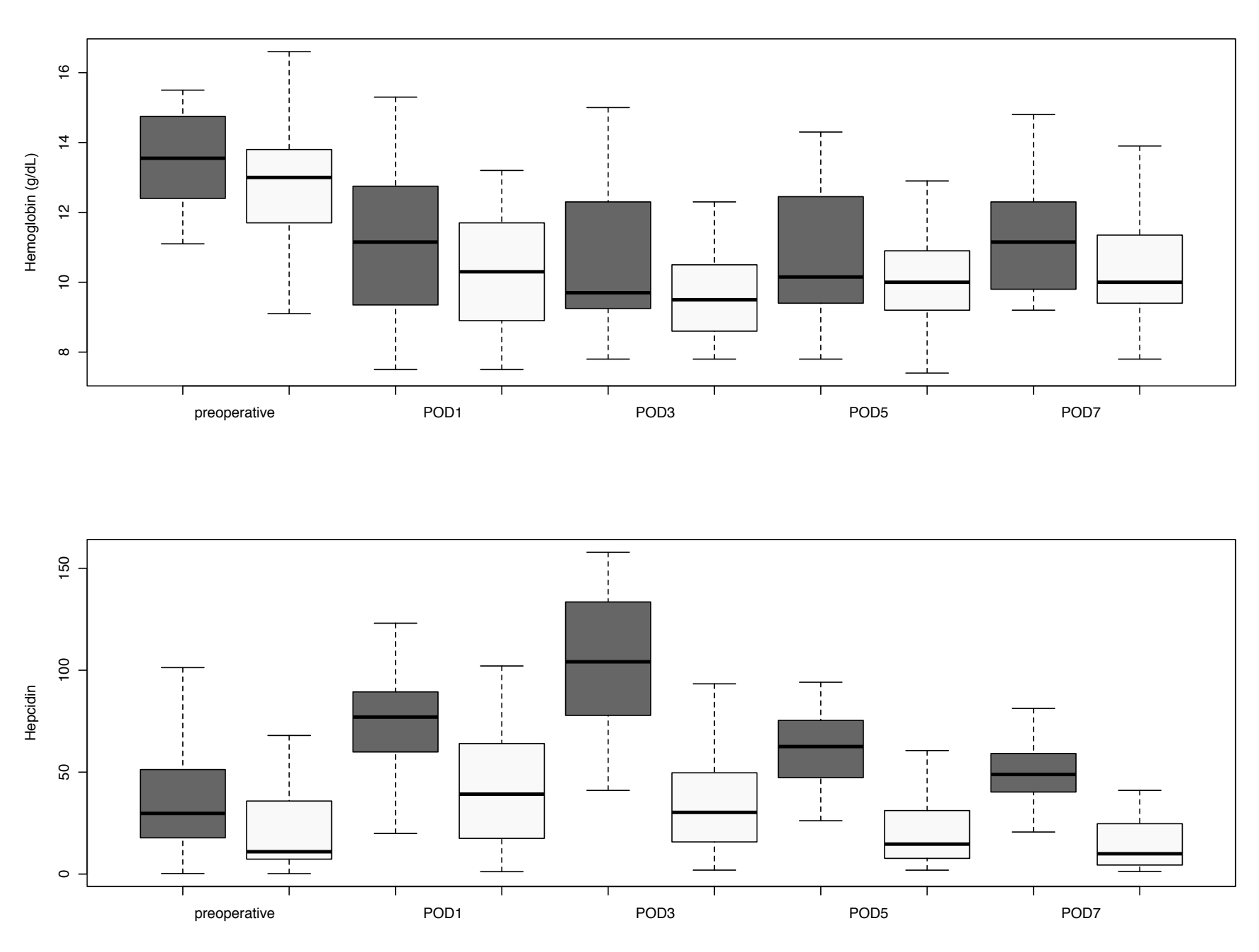

\section{$\underline{\text { Results }}$}

Both groups were comparable regarding patients' baseline characteristics, type of surgery, pathology analyses and postoperative complications.

Iron supplementation was associated with an increase of I.2 $\mathrm{g} / \mathrm{dL}$ of $\mathrm{Hb}$ level $(\mathrm{p}=0.04)$ and higher hematocrit level (34.7 (30.9-36.6) vs 30.4 (29.0-33.5); $\mathrm{P}=0.02$ ) at $\mathrm{POD} 7$.

Hepcidin level was also higher after iron supplementation (50ng/l [30,4-I34] vs 8,4ng/l [4,4-25] ; $\mathrm{P}<0.000 \mathrm{I}$ ) whereas IL-6 level did not differ across groups. At POD 7, the median ferritin level was I307ug/l (1099-167I) vs 24lug/l (174-497) in the iron group $(p<0.00 \mathrm{I})$. Finally, lenght of hospitalisation and rate of blood transfusion were comparable.

\section{Material and methods}

The HepciFer trial was a single-center, randomized, placebo-controlled, double-blinded clinical trial (RCT) in 50 patients undergoing liver surgery. Adult patients undergoing elective liver surgery were screened and included from September 2015 to August 2016. According to the randomization process, patients received either ferric carboxymaltose $(15 \mathrm{mg} / \mathrm{Kg}$, maximum $\mathrm{Ig}$ ) or placebo 4 hours after surgery. Primary outcome assessed the difference of plasma $\mathrm{Hb}$ levels between the two groups seven days after surgery. Secondary outcomes included plasma level of Hepcidin and iron status at different time points after surgery, length of hospitalization and incidence of blood transfusion.
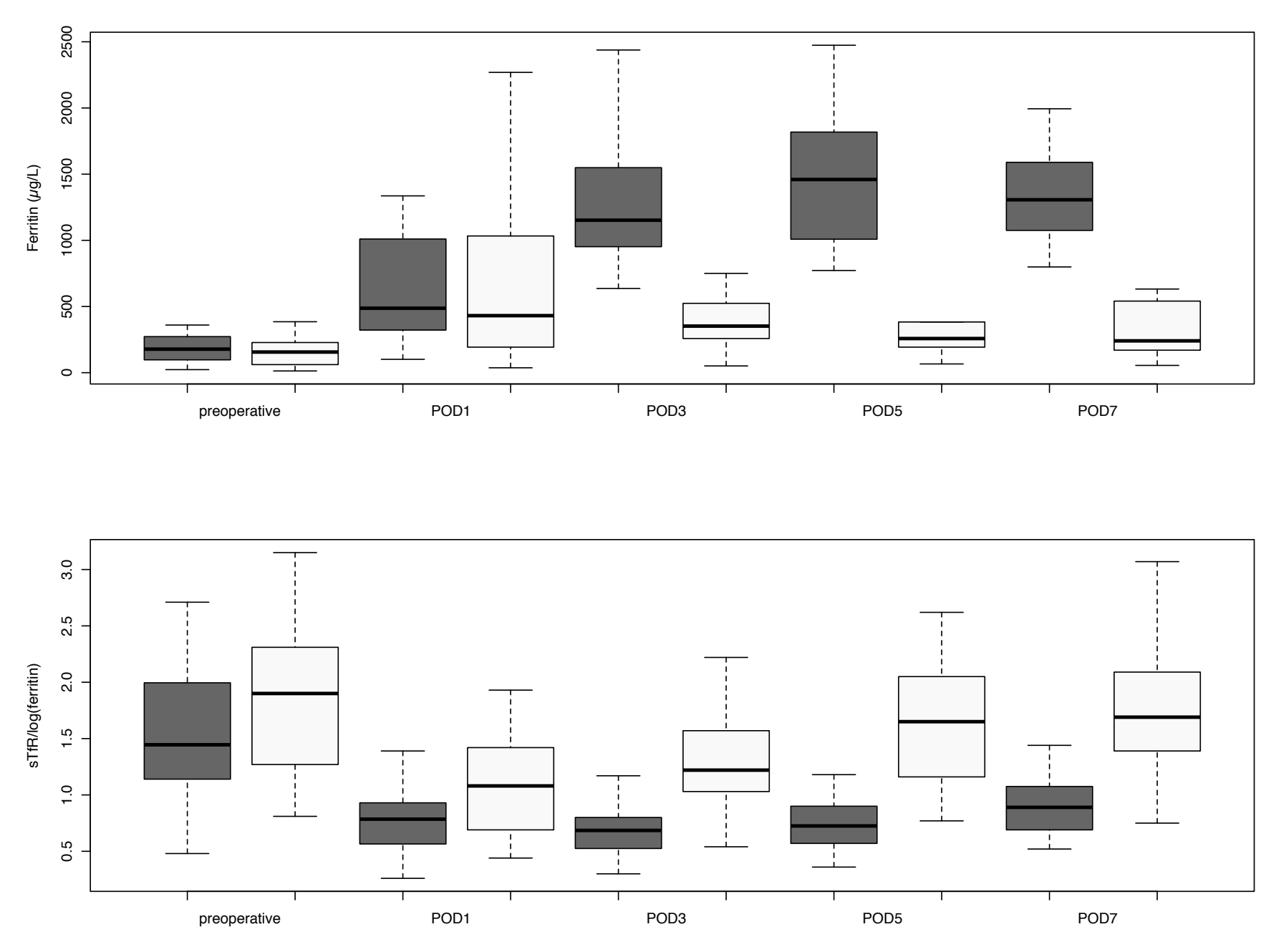

\section{Conclusion}

This study is to our knowledge the first RCT to evaluate the impact of IV iron administration in the postoperative period after liver surgery. Our findings suggest that IV iron supplementation increase $\mathrm{Hb}$ and $\mathrm{Hct}$ level at POD 7 by treating the FID. In addition, this trial adds new knowledge regarding the physiopathology of iron metabolism and Hepcidin kinetics and thus, provides a better understanging of mechanisms involved in FID during postoperative period after liver surgery. 